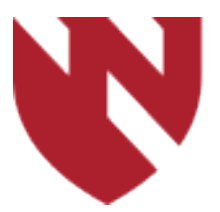

\title{
An Uncommon Adverse Effect of a Common Drug-DRESS Syndrome-not just skin deep!
}

Alamelu Udayappan

University of Nebraska Medical Center

Tell us how you used this information in this short survey.

Follow this and additional works at: https://digitalcommons.unmc.edu/gmerj

Part of the Higher Education Commons, and the Pediatrics Commons

\section{Recommended Citation}

Udayappan, A. An Uncommon Adverse Effect of a Common Drug-DRESS Syndrome-not just skin deep!. Graduate Medical Education Research Journal. 2019 Dec 13; 1(1).

https://digitalcommons.unmc.edu/gmerj/vol1/iss1/9

This Case Report is brought to you for free and open access by DigitalCommons@UNMC. It has been accepted for inclusion in Graduate Medical Education Research Journal by an authorized editor of DigitalCommons@UNMC. For more information, please contact digitalcommons@unmc.edu. 


\title{
An Uncommon Adverse Effect of a Common Drug-DRESS Syndrome-not just skin deep!
}

\author{
Abstract \\ Drug Rash with Eosinophilia and Systemic Symptoms (DRESS) Syndrome is a rare, life-threatening \\ hypersensitivity reaction that causes skin exfoliation, hematological abnormalities, lymphadenopathy, and \\ extensive internal organ involvement. Characterized by a long latency period, this unusual side effect of \\ aromatic anti-epileptic drugs, as well as many common drugs cause a mortality rate of up to $10 \%$ if left \\ untreated. In the absence of a well-established therapy, primary and secondary prevention have a key role \\ in the management of DRESS Syndrome. Herewith presenting a case of an adolescent girl with rashes \\ and systemic symptoms, the cause of which, on obtaining a detailed history and step wise investigations \\ was diagnosed as DRESS Syndrome.

\section{Creative Commons License}

\section{cc) (i) $(9$}

This work is licensed under a Creative Commons Attribution-Noncommercial-No Derivative Works 4.0 License. 


\section{An Uncommon Adverse Effect of a Common Drug-DRESS Syndrome-Not Just Skin Deep!}

\section{Alamelu Udayappan}

${ }^{1}$ University of Nebraska Medical Center, Department of Pediatrics

https://doi.org/10.32873/unmc.dc.gmerj.1.1.009

\begin{abstract}
Drug Rash with Eosinophilia and Systemic Symptoms (DRESS) Syndrome is a rare, life-threatening hypersensitivity reaction that causes skin exfoliation, hematological abnormalities, lymphadenopathy, and extensive internal organ involvement. Characterized by a long latency period, this unusual side effect of aromatic anti-epileptic drugs, as well as many common drugs cause a mortality rate of up to $10 \%$ if left untreated. In the absence of a well-established therapy, primary and secondary prevention have a key role in the management of DRESS Syndrome. Herewith presenting a case of an adolescent girl with rashes and systemic symptoms, the cause of which, on obtaining a detailed history and step wise investigations was diagnosed as DRESS Syndrome.
\end{abstract}

\section{Introduction}

A skin rash with hematological abnormalities in an adolescent girl poses several differential diagnoses. One under-recognized etiology of skin changes with internal organ involvement in this age group is Drug Rash with Eosinophilia with Systemic Symptoms (DRESS Syndrome). DRESS Syndrome is diagnosed by a process of elimination, ruling out the more common conditions including Systemic Lupus Erythematosus, Kawasaki, and Steven Johnson Syndrome in addition to confirming the diagnosis using the RegiSCAR criteria. ${ }^{1}$ There is paucity of data on DRESS syndrome and thus many go undiagnosed or are improperly managed. An early diagnosis of DRESS Syndrome is key to successful treatment and reduction in mortality. Herewith presenting a case of DRESS syndrome with classic clinical and biochemical features whom had quick recovery following withdrawal of the suspected drug along with short course of steroid therapy. Consent was obtained from the patient to use her information and images for educational purposes.

\section{Case}

A 17 year old, developmentally normal adolescent, presented with acute onset abdominal pain, fever and vomiting. She soon developed maculopapular rashes beginning with face (Figure 1) and extremities (Figure 2) that later became generalized with itching. There was no similar episode in the past and no contributory family history. Positive examination findings included non-tender significant cervical lymphadenopathy, non-palpable maculopapular rashes over the extensor aspects of the limbs and face with exfoliation of skin over the extremities, bilateral pitting and pedal edema (Figure 3). Abdominal examination revealed moderate hepatomegaly and stable vital signs.

Baseline investigations were done that revealed a total hemoglobin count of $9.9 \mathrm{mg} / \mathrm{dl}$, total white cell count of $15,400 \mathrm{cu} / \mathrm{mm}$ with eosinophilia of $27 \%$ and a normal platelet count. Her absolute eosinophil count was $4,000 \mathrm{cu} / \mathrm{mm}$ and her Erythrocyte Sedimentation Rate (ESR) was moderately elevated at $32 \mathrm{~mm} / \mathrm{hr}$. In view of hepatomegaly, liver function tests were done that showed an elevated SGOT of $90 \mathrm{U} / \mathrm{L}$, SGPT of $86 \mathrm{U} / \mathrm{L}$ with normal bilirubin and alkaline phosphatase levels. She had trace proteins in her urine. Her urine spot protein creatinine ratio was less than 2 , normal Complement 3 (C3), and Complement 4 (C4) levels with Antinuclear Antibodies (ANA) negative. An echocardiogram demonstrated no pertinent findings. On probing her past history, she was recently evaluated by a private practitioner and diagnosed with probable complex partial seizures for which she was started on carbamazepine at the dose of $2 \mathrm{mg} /$ $\mathrm{kg} 3$ times a day over the last 4 weeks. As she had skin involvement with eosinophilia and hepatic involvement, Drug Reaction with Eosinophilia and Systemic Symptoms (DRESS) Syndrome was suspected. The offending drug was immediately stopped and replaced with Levetiracetam. A skin biopsy was performed which demonstrated eosinophilia along with atypical lymphocytes, confirming the diagnosis of DRESS Syndrome. As she had a prolonged course of illness, she was also treated with a short course steroid therapy. Within a week of therapy, she experienced complete clinical and biochemical remission.

\section{Discussion}

With the initial presenting complaints of acute onset fever and rash, a simple viral exanthematous illness was our first

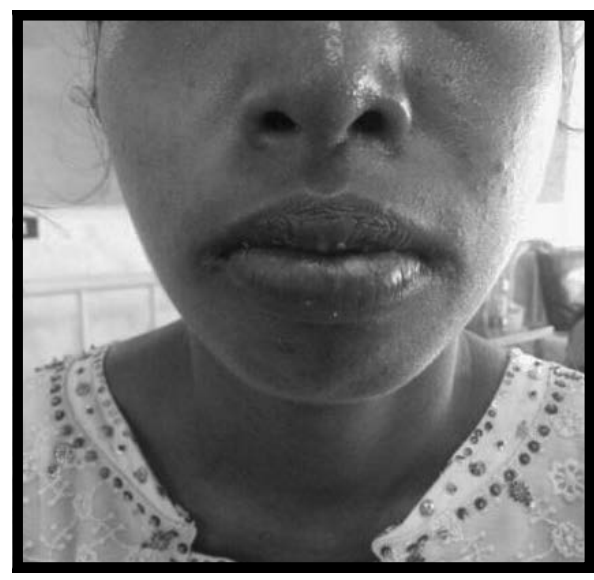

Figure 1. Cutaneous maculopapular eruption with exfoliate dermatitis and facial edema with cheilitis noted.

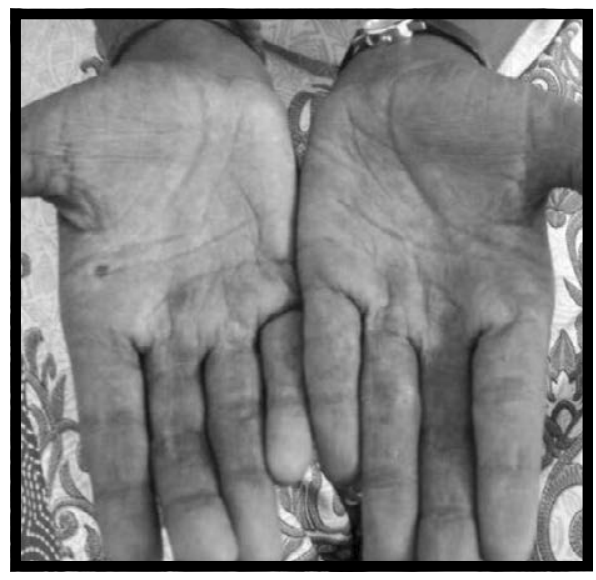

Figure 2. Maculopapular rash seen affecting the palms.

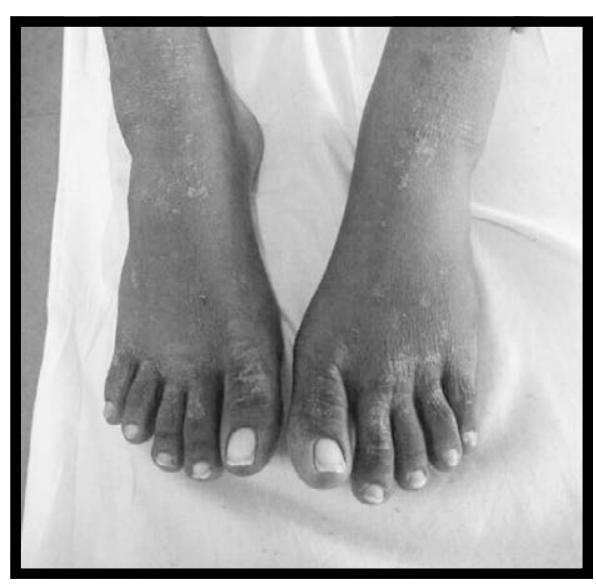

Figure 3. Maculopapular rash with desquamation and pedal edema affecting the lower extremities. 
outpatient diagnosis. But the prolonged course of illness that was not resolving with medical management and the characteristic maculopapular rash with itching and exfoliation urged us to look for an alternative diagnosis.

In the general population, the first course of action for an adolescent girl presenting with rash, elevated ESR, and trace protein in urine is to rule out Systemic Lupus Erythematosus (SLE). A rash in SLE is more often a malar or butterfly type which varies from an erythematous blush to thickened epidermis to scaly patches which may be photosensitive and extend to all sun-exposed areas. ${ }^{2}$ Our child did not fit into the classical Revised Classification Criteria for Systemic Lupus Erythematosus clinically as well as biochemically because her ANA was negativethus we probed further into evaluating this young girl.

On further examination, the presence of rash, edema of feet, and non-tender unilateral lymph node cervical enlargement, Kawasaki disease was considered. However, to diagnose Kawasaki disease, at least 4 of the principle clinical features, including the full epidermal peeling-periungual peeling, changes in extremities erythema of palms, soles, polymorphous exanthema, bilateral bulbar conjunctival injection without exudate erythema, lip cracking, and strawberry tongue are essential. ${ }^{3}$ But our patient had more of a monomorphous rash, with exfoliation only at the tips of digits (Figure 4) and therefore did not fit into the diagnostic criteria. We proceeded with an echocardiogram to rule out incomplete forms of Kawasaki disease as the child was in the adolescent age group with moderately elevated ESR. The echocardiogram was normal.

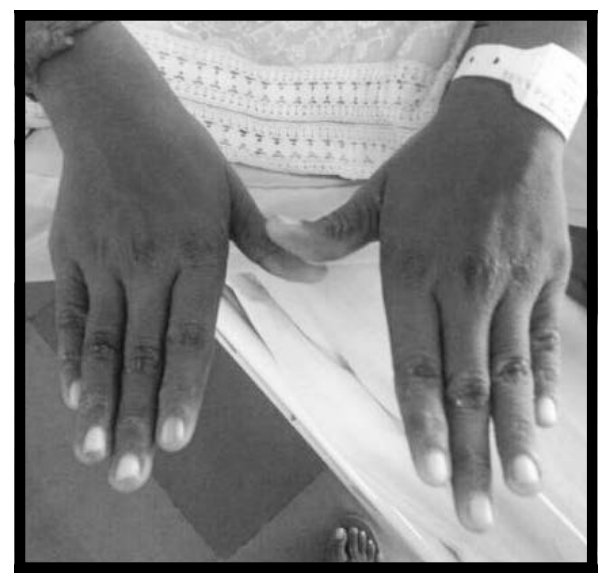

Figure 4. Monomorphous rash and with exfoliation affecting the digits.

Due to recent anti-seizure medication and her clinical picture, the possibility of Steven Johnson Syndrome (SJS) was considered. SJS can occur 2 to 6 weeks following initiation of similar anticonvulsant medication and present with an eosinophilic blood picture. ${ }^{4}$ However, the patient's rash, beginning from face and extremities, and hepatic involvement combined with absence of mucosal involvement were uncharacteristic of SJS.

In conclusion, we narrowed our diagnosis to DRESS Syndrome due to the constellation of findings consisting of fever, rash following carbamazepine intake in the recent past requiring hospitalization, eosinophilia and hepatic involvement. In addition, the patient presented with findings that satisfied the RegiSCAR criteria for the diagnosis of DRESS Syndrome. ${ }^{1}$ A skin biopsy showing increased eosinophil count along with atypical lymphocytes reaffirmed our diagnosis. Furthermore, mere withdrawal of the offending drug and a short course of steroids led to a complete clinical and biochemical resolution.

\section{Conclusion}

Diagnosis of DRESS Syndrome can be difficult due to the variable presentations of the syndrome and is often obtained only after exclusion of commoner causes. In addition, the long latency period following initiation or after stopping the medication creates difficulties in diagnosis. This report is of clinical significance due to paucity of data DRESS Syndrome as many cases go undiagnosed or result in an alternate diagnosis. This case was presented to highlight the fact that a clinical suspicion of the above is essential for appropriate management and prevention of mortality.

\section{References}

1 Chiou CC, Yang LC, Hung SI, et al. Clinicopathological features and prognosis of drug rash with eosinophilia and systemic symptoms: a study of 30 cases in Taiwan. J Eur Acad Dermatol Venereal, 2008; 22:1044-1049.

2 Das NK, Dutta RN, Sengupta SR. Skin lesions in lupus erythematosus: a marker of systemic involvement. Indian J Dermatol. 2011; 56(5):537-540.

3 Sathavahana C, Kumar V, Koteswara R, Sudha R. Kawasaki disease (syndrome)-our experience. Indian $J$ Otolaryngol Head Neck Surg. 2007; 59(1):68-70.

4 Rzany B, Correia O, Kelly JP, et al. Risk of StevensJohnson Syndrome and toxic epidermal necrolysis during first weeks of antiepileptic therapy: a case-control study. Study Group of the International Case Control Study on Severe Cutaneous Adverse Reactions. Lancet. 1999; 353: 2190-2194. 